Research

\title{
Genetic correlates of longevity and selected age-related phenotypes: a genome-wide association study in the Framingham Study
}

\author{
Kathryn L Lunetta1,3, Ralph B D'Agostino Sr1,5, David Karasik ${ }^{4}$, \\ Emelia J Benjamin1,2, Chao-Yu Guo1,2, Raju Govindaraju1,2, Douglas P Kiel ${ }^{4}$, \\ Margaret Kelly-Hayes ${ }^{1,2}$, Joseph M Massaro',5, Michael J Pencina1,5, \\ Sudha Seshadri1,2 and Joanne M Murabito*1,2
}

\begin{abstract}
Address: ${ }^{1}$ The National Heart Lung and Blood Institute's Framingham Heart Study, Framingham, MA, USA, ${ }^{2}$ Section of General Internal Medicine and the Departments of Neurology, Cardiology, and Preventive Medicine, Boston University School of Medicine, Boston, MA, USA, ${ }^{3}$ Department of Biostatistics, Boston University School of Public Health, Boston, MA, USA, ${ }^{4}$ Hebrew Senior Life Institute for Aging Research and Harvard Medical School, Boston, MA, USA and ${ }^{5}$ Statistics and Consulting Unit, Department of Mathematics, Boston University, Boston, MA, USA

Email: Kathryn L Lunetta - klunetta@bu.edu; Ralph B D'Agostino - ralph@bu.edu; David Karasik - karasik@hrca.harvard.edu; Emelia J Benjamin - emelia@bu.edu; Chao-Yu Guo - chaoyu@bu.edu; Raju Govindaraju - drgraju@bu.edu; Douglas P Kiel - kiel@hrca.harvard.edu; Margaret Kelly-Hayes - mkhayes@bu.edu; Joseph M Massaro - jmm@bu.edu; Michael J Pencina - mpencina@bu.edu; Sudha Seshadri - suseshad@bu.edu; Joanne M Murabito* - murabito@bu.edu

* Corresponding author
\end{abstract}

Published: 19 September 2007

BMC Medical Genetics 2007, 8(SuppI I):SI3 doi:I0.II86/I47I-2350-8-SI-SI3

This article is available from: http://www.biomedcentral.com/I47I-2350/8/SI/SI3

(c) 2007 Lunetta et al; licensee BioMed Central Ltd.

This is an open access article distributed under the terms of the Creative Commons Attribution License (http://creativecommons.org/licenses/by/2.0), which permits unrestricted use, distribution, and reproduction in any medium, provided the original work is properly cited.

\begin{abstract}
Background: Family studies and heritability estimates provide evidence for a genetic contribution to variation in the human life span.

Methods: We conducted a genome wide association study (Affymetrix I00K SNP GeneChip) for longevity-related traits in a community-based sample. We report on 5 longevity and aging traits in up to I345 Framingham Study participants from 330 families. Multivariable-adjusted residuals were computed using appropriate models (Cox proportional hazards, logistic, or linear regression) and the residuals from these models were used to test for association with qualifying SNPs (70, 987 autosomal SNPs with genotypic call rate $\geq 80 \%$, minor allele frequency $\geq 10 \%$, Hardy-Weinberg test $p \geq 0.00 I$ ).

Results: In family-based association test (FBAT) models, 8 SNPs in two regions approximately $500 \mathrm{~kb}$ apart on chromosome I (physical positions 73,091,610 and 73, 527,652) were associated with age at death ( $\mathrm{p}$-value $<10^{-5}$ ). The two sets of SNPs were in high linkage disequilibrium (minimum $r^{2}=0.58$ ). The top 30 SNPs for generalized estimating equation (GEE) tests of association with age at death included rsI0507486 ( $p=0.0001)$ and rs4943794 $(p=0.0002)$, SNPs intronic to FOXOIA, a gene implicated in lifespan extension in animal models. FBAT models identified 7 SNPs and GEE models identified 9 SNPs associated with both age at death and morbidity-free survival at age 65 including rs 2374983 near PONI. In the analysis of selected candidate genes, SNP associations (FBAT or GEE p-value $<0.0 \mathrm{I}$ ) were identified for age at death in or near the following genes: FOXOIA, GAPDH, KL, LEPR, PONI, PSENI, SOD2, and WRN. Top ranked SNP associations in the GEE model for age at natural menopause included rs6910534 ( $p=0.00003)$ near FOXO3a and rs375I59I ( $p=0.00006)$ in CYPI9AI. Results of all longevity phenotype-genotype associations for all autosomal SNPs are web posted at http://www.ncbi.nlm.nih.gov/projects/gap/cgi-bin/study.cgi?id=phs000007.
\end{abstract}


Conclusion: Longevity and aging traits are associated with SNPs on the Affymetrix I00K GeneChip. None of the associations achieved genome-wide significance. These data generate hypotheses and serve as a resource for replication as more genes and biologic pathways are proposed as contributing to longevity and healthy aging.

\section{Background}

Genetic factors associated with human longevity and healthy aging remain largely unknown. Heritability estimates of longevity derived from twin registries and large population-based samples suggest a significant but modest genetic contribution to the human lifespan (heritability 15 to $30 \%$ ) [1-4]. However, genetic influences on lifespan may be greater once an individual achieves age 60 years [5]. Moreover, the reported magnitude of the genetic contribution to other important aspects of aging such as healthy physical aging (wellness)[6], physical performance $[7,8]$, cognitive function [9], and bone aging [10] are much larger. Both exceptional longevity and a healthy aging phenotype have been linked to the same region on chromosome $4[11,12]$, suggesting that although longevity per se and healthy aging are different phenotypes, they may share some common genetic pathways.

A number of potential candidate genes in a variety of biological pathways have been associated with longevity in model organisms. Genes involved in the regulation of DNA repair and genes in the evolutionarily conserved insulin/insulin-like growth factor signaling pathway $[13,14]$ are emerging as holding great promise in the future elucidation of the underlying physiology controlling lifespan. Many of these genes have human homologs and thus have potential to provide insights into human longevity [15-20]. Although numerous candidate genes have been proposed, studies in humans are limited and initial findings often fail replication [21,22]. More recently genome-wide association studies (GWAS) have become feasible and offer a more comprehensive and untargeted approach to detect genes with modest phenotypic effects that underlie common complex conditions [23].

We had the opportunity to use the Framingham Heart Study (FHS) Affymetrix 100K SNP genotyping resource for a GWAS of longevity and aging-related phenotypes. The FHS offers the unique advantage of a longitudinal family-based community sample with participants who have been well-characterized throughout adulthood with respect to prospectively ascertained risk factors and diseases and continuously followed until death. We report several strategies for $100 \mathrm{~K}$ SNP associations: 1) a simple low p-value SNP ranking strategy; 2) SNP selection due to associations with more than one related phenotype; and 3) SNP associations within candidate genes and regions previously reported to be associated with longevity in model organisms or humans.

\section{Methods \\ Study sample}

The genotyped study sample is comprised of 1345 Original cohort $(\mathrm{n}=258)$ and Offspring $(\mathrm{n}=1087)$ participants who are members of the 330 largest FHS families. The Overview [24] provides further details of this sample. With respect to aging and longevity traits, 149 deaths occurred at mean age at death of 83 years (range 46 to 99 years) and 713 participants achieved age 65 years or greater. The Boston University Medical Center Institutional Review Board approved the examination content of Original Cohort and Offspring examinations. All participants provided written informed consent at every examination including consent for genetic studies.

\section{Longevity and aging phenotype definitions and residual creation}

Age at death

Both the Original Cohort and the Offspring Cohort remain under continuous surveillance and all deaths that occurred prior to January 1, 2005 were included in this study. Deaths were identified using multiple strategies including routine participant contact for research examinations or health history updates, surveillance at the local hospital, search of obituaries in the local newspaper, and if needed through use of the National Death Index. Death certificates were routinely obtained and all hospital and nursing home records prior to death and autopsy reports (if performed) were requested. In addition, if there was insufficient information to determine a cause of death, the next of kin were interviewed by a senior investigator. All records pertinent to the death were reviewed by an endpoint panel comprised of three senior investigators. The date and cause of death (classified as due to coronary heart disease, stroke, other cardiovascular disease [CVD], cancer, other causes, or unknown cause) was recorded.

Cox proportional hazards models were used to generate martingale residuals using the PHREG procedure in SAS to perform the regression analysis of survival time from age at study entry to age at death. Models were sex-specific and adjusted for 1) birth cohort and 2) birth cohort, education, current smoking status (yes/no), obesity (body mass index $\geq 30 \mathrm{~kg} / \mathrm{m}^{2}$ ), hypertension (blood pressure $\geq 140 / 90 \mathrm{mmHg}$ or on antihypertensive treatment), elevated cholesterol (cholesterol > $239 \mathrm{mg} / \mathrm{dL}$ ), diabetes 
(fasting blood sugar $\geq 126 \mathrm{mg} / \mathrm{dL}$, random blood sugar of $\geq 200 \mathrm{mg} / \mathrm{dL}$, or use of insulin or oral hypoglycemic agents) and comorbidity defined as CVD and cancer. Birth cohort was defined as a categorical variable for all regression models with the following categories based on year of birth: birth year prior to 1900,1900 to 1909,1910 to 1919,1920 to 1929,1930 to 1939,1940 to 1949 , and 1950 and later. All covariates were measured at study entry. Residuals from Original Cohort and Offspring participants were pooled.

\section{Morbidity-free survival at age 65 years}

Morbidity-free survival was defined as achieving age 65 years free of CVD, dementia, and cancer. CVD events included angina pectoris, coronary insufficiency, myocardial infarction, heart failure, stroke, transient ischemic attack (TIA), intermittent claudication and coronary or CVD death. Suspected CVD events were reviewed by a panel of three investigators who adjudicated events using previously established criteria in place since study inception [25]. A separate panel of study neurologists determined the presence of stroke or TIA and a team of at least one neurologist and one neuropsychologist determined the presence of dementia. Two independent reviewers examined records for all cancers, and the vast majority of cancer cases were microscopically confirmed with pathology reports.

Logistic regression models were used to generate deviance residuals. Models were sex-specific and adjusted for 1) birth cohort and 2) birth cohort, education, current smoking status, obesity, hypertension, elevated cholesterol, and diabetes. Covariates were defined as above for age at death. All covariates were measured at the examination closest to the participant attaining age 65 years using a 5 year window around age 65 years. Residuals from Original Cohort and Offspring participants were pooled.

\section{Age at natural menopause}

Natural menopause occurred after a woman had ceased menstruating naturally for one year and the age at natural menopause was the self-reported age at last menstruation. Mean age at natural menopause was similar in Original Cohort and Offspring women and the distribution of naturally menopausal ages in women in the 330 FHS families was similar to that of women in all 1643 FHS families $[26,27]$. The mean age at natural menopause in women in the $100 \mathrm{~K}$ sample was 50.2 years (range 38 to 57 years) in Original Cohort women and 49.1 years (range 29 to 60 years) in Offspring women.

Crude age at natural menopause and standardized residuals from multiple linear regressions in SAS [28] that adjusted age at natural menopause for covariates of interest were used as traits for analysis. Covariates were obtained at all attended examinations prior to the onset of menopause and included mean number of cigarettes smoked per day, mean body mass index, parity ( 0 versus 1 or more live births), and generation (Original Cohort vs. Offspring).

\section{Walking speed}

Walking speed was measured on Original Cohort participants at examination 27 (January 2002 through December 2003, mean age of Original Cohort at exam 27: 86.7 years) and Offspring participants attending an ancillary study to examination 7 (1999 to 2004, mean age at exam: 62.0 years). Trained technicians timed participants walking at their normal pace on a four meter course twice and subsequently asked participants to repeat the course walking at a rapid pace. The mean timed fast walk among Offspring participants in the $100 \mathrm{~K}$ genotyping sample was 2.44 seconds (standard deviation 0.89 ). The timed fast walk was used for analysis. Sex-specific linear regression was used to generate residuals adjusted for age and height measured at the time of the walk.

\section{Biologic age by osseographic scoring system}

An osseographic scoring system (OSS) was applied to hand radiographs obtained on original cohort (1967 to 1969, mean age 58.7 years) and offspring participants (1992 to 1993, mean age 51.6 years) [10]. Biologic age was then defined as the standardized residual between the OSS predicted age and the actual age. Biologic age defined by this system predicted mortality $[10,29]$, was very heritable $\left(h^{2}=0.57 \pm 0.06\right)$, and a genome-wide linkage analysis was performed with LOD scores $>1.8$ present on chromosomes 3q, 11p, 16q, and 21q [10]. Sex- and cohort-specific ranked residuals generated from linear regression of age on log-OSS adjusted for height, body mass index, menopause, and estrogen therapy, were used for analysis.

\section{Genotyping}

Affymetrix 100K SNP GeneChip genotyping and the Marshfield STR genotyping performed by the Mammalian Genotyping Service http://research.marshfieldclinic.org/ genetics are described in the Overview paper [24].

\section{Statistical analysis}

The statistical methods for genome-wide linkage and association analyses are described in the Overview [24].

\section{Association}

All residual traits described above as well as the additional traits listed in Table 1 were computed using Cox proportional hazards with martingale residuals for survival traits, logistic regression with deviance residuals for dichotomous traits, and linear regression with standard residuals for quantitative traits. The full set of FHS participants with 
the phenotype were used to create the residuals. The residuals were used to test for association between the genotyped subset of individuals and the SNPs using additive family-based association test (FBAT) and generalized estimating equations (GEE) models as described in the Overview [24]. A total of 70,987 autosomal SNPs met the criteria of genotypic call rate $\geq 80 \%$, minor allele frequency $\geq 10 \%$, Hardy-Weinberg test $\mathrm{p} \geq 0.001$, and $\geq 10$ informative families for FBAT. The number of tests with an FBAT $\mathrm{p}<0.001, \mathrm{p}<0.0001$, and $\mathrm{p}<0.00001$ for all phenotypes was similar to what would be expected under the assumptions that the 70,987 tested SNPs were independent and there were no true associations. The GEE tests tended to give an excess of very small p-values over what would be expected under these assumptions.

\section{SNP prioritization}

We used several strategies to prioritize SNPs associated with longevity and aging traits. First, we used an untargeted approach whereby the top 50 SNP associations ranked according to the strength of the p-value for each trait were examined. Next, we explored the consistency of SNP associations across related sets of traits chosen a priori (trait set one: age at death and morbidity-free survival at age 65 years; trait set two: biologic age and walking speed). Trait set one was chosen based upon linkage data in humans demonstrating that both longevity and a healthy aging trait were linked to the same region on chromosome 4 raising the hypothesis that the two phenotypes may share common genetic pathways $[11,12]$. The traits in set two reflect aging with good physical functioning and thus we postulated that biologic age and walking speed may have genetic variants in common. We also investigated SNP associations in candidate genes and regions reported to be associated with longevity identified from established databases including NCBI [14] using the search term "longevity" and the Science of Aging Knowledge Environment genes/intervention database http:// sageke.sciencemag.org/cgi/genesdb[30] choosing genes potentially related to lifespan in humans.

The SNPs were annotated using the UCSC genome browser tables using the May 2004 assembly http:// genome.ucsc.edu/[31,32]. All genes within $60 \mathrm{~kb}$ of the top ranked SNPs were identified.

\section{Results}

The longevity and aging traits available in the FHS $100 \mathrm{~K}$ SNP resource are listed in Table 1. In this report, we consider only five of the traits listed in Table 1: multivariableadjusted age at death, morbidity-free survival at age 65 years, age at natural menopause, walking speed, and biologic age by OSS. These traits include a pooled sample of Original Cohort and Offspring participants, with the exception of walking speed, which is reported in Off- spring participants only. Details of the sample size and covariate adjustment for each trait are provided in Table 1 .

For each of the five phenotypes, Table $2 \mathrm{a}$ and $2 \mathrm{~b}$ provides the top five SNPs ranked in order by lowest $p$-value for the GEE and FBAT models (all associations can be viewed on the web http://www.ncbi.nlm.nih.gov/projects/gap/cgibin/study.cgi?id=phs000007). If multiple SNPs in linkage disequilibrium $\left(\mathrm{LD} \mathrm{r}^{2}>0.80\right)$ were included in the top 5, additional SNPs were included until a set of 5 independent associations were listed. Eight SNPs on chromosome 1 were associated with age at death in the FBAT analysis; all with $\mathrm{p}$-value $<10^{-4}$ and two with p-value $<10^{-5}$. The 8 SNPs consisted of two sets of SNPs (rs10493513, rs10493514, rs6689491, rs6657082, rs1405051) and (rs10493515, rs10493518, rs10493517), clustered in two regions approximately $500 \mathrm{~kb}$ apart. There was exceptionally high LD across this $500 \mathrm{~kb}$ region: the minimum $\mathrm{r}^{2}$ between pairs of the eight SNPs was 0.58. The nearest genes in this region existing in public databases were $>500$ $\mathrm{kb}$ from any of these SNPs [31,32].

There were several additional associations not listed in Table $2 \mathrm{a}$ and $2 \mathrm{~b}$ that were of interest. For age at death in the GEE analysis, SNP associations ranked numbers 9 and 13 were rs 10507486 (p-value 0.000128) and rs4943794 (p-value 0.000277), both are intronic FOXO1A SNPs. For age at natural menopause, top ranked SNP associations in the GEE model included number 11, rs6910534 ( $\mathrm{p}=$ 0.00003 ) near FOXO3a and number 18, rs3751591 (p = $0.00006)$ in CYP19A1.

Table $2 \mathrm{c}$ presents the LOD scores $\geq 2.0$ and the corresponding 1.5-LOD support interval from genome-wide linkage for the three quantitative aging traits. None of the regions overlapped with SNPs associated with these aging traits in the FBAT and GEE analyses. Of note for biologic age by OSS the linkage peak on chromosome 21 confirmed a prior Framingham Study report using a genomewide scan with 401 microsatellite markers [10].

Table 3 provides all SNP associations with a GEE or FBAT $\mathrm{p}<0.01$ for both traits within the two pairs of related traits. For age at death and morbidity-free survival at age 65 years, FBAT models identified 7 SNPs and GEE models identified 9 SNPs associated with both traits including rs2374983 near PON1 (Tables 3a and 3b). For biologic age by OSS and walking speed, 13 SNPs in FBAT models and 6 SNPs in GEE models were associated with both traits (Tables $3 \mathrm{c}$ and $3 \mathrm{~d}$ ).

We identified from the literature 79 potential candidate genes and regions associated with longevity (see Additional file 1 for listing). Of these, 12 genes had no SNPs and 67 genes had 1 to 45 SNPs within $60 \mathrm{~kb}$ of the gene 
Table I: Aging and Longevity Phenotypes for Framingham Heart Study I00K Project

\begin{tabular}{|c|c|c|c|c|}
\hline \multicolumn{5}{|c|}{ Exam cycle(s) } \\
\hline $\begin{array}{l}\text { Phenotype Subgroup } \\
\text { Trait (variable name on the } \\
\text { website*) }\end{array}$ & Number of Traits & $\mathbf{N}\left(\mathbf{M V}^{* *}\right)$ & $\begin{array}{l}\text { Offspring / Original } \\
\text { Cohort }\end{array}$ & Adjustment \\
\hline \multicolumn{5}{|c|}{ Survival Traits: Cox regression } \\
\hline $\begin{array}{l}\text { Survival } \\
\text { - Age at death (I. deathageX, } 2 \text {. } \\
\text { deathageMV) }\end{array}$ & 2 & $1345(1166)$ & $\begin{array}{l}\text { Cohort \& Offspring } \\
\text { pooled }\end{array}$ & $\begin{array}{l}\text { Cox regression } \\
\text { Sex-specific } \\
\text { I. birth cohort } \\
\text { 2. multivariable adjusted for birth cohort, education, } \\
\text { smoking, obesity (BMI } \geq 30 \text { ), CVD risk factors, co-morbidity } \\
\text { measured at exam I }\end{array}$ \\
\hline
\end{tabular}

\begin{tabular}{|c|c|c|c|c|}
\hline \multicolumn{5}{|c|}{ Categorical traits: Logistic regression } \\
\hline $\begin{array}{l}\text { - Survival past the ALE (I. } \\
\text { deathpastALEX, 2. deathpastALEMV) }\end{array}$ & 2 & $1345(1166)$ & $\begin{array}{l}\text { Cohort \& Offspring } \\
\text { pooled }\end{array}$ & $\begin{array}{l}\text { Logistic regression } \\
\text { Sex-specific } \\
\text { I. birth cohort } \\
\text { 2. multivariable adjusted for birth cohort, education, } \\
\text { smoking, obesity (BMI } \geq 30 \text { ), CVD risk factors, co-morbidity } \\
\text { measured at exam I }\end{array}$ \\
\hline $\begin{array}{l}\text { Morbidity-free survival (free of } \\
\text { CVD, cancer and dementia) } \\
\text { - At age } 65 \text { years (I. } \\
\text { morbidityfree65X, } 2 \text {. } \\
\text { morbidityfree65MVX) }\end{array}$ & 2 & $558(558)$ & $\begin{array}{l}\text { Cohort \& Offspring } \\
\text { pooled, exams closest to } \\
\text { age } 65 \text { years }\end{array}$ & $\begin{array}{l}\text { Logistic regression } \\
\text { Sex-specific } \\
\text { I. birth cohort } \\
\text { 2. multivariable adjusted for birth cohort, education, } \\
\text { smoking, obesity, CVD risk factors measured at exam closest } \\
\text { to age } 65 \text { years (within a } 5 \text { year horizon) }\end{array}$ \\
\hline
\end{tabular}

\begin{tabular}{|c|c|c|c|c|}
\hline \multicolumn{5}{|c|}{ Quantitative Traits: Linear regression } \\
\hline $\begin{array}{l}\text { Reproductive Aging } \\
\text { - Age at natural menopause (I. } \\
\text { menoageX, 2. menoageMVX) }\end{array}$ & 2 & 438 (378) & $\begin{array}{l}\text { Cohort \& Offspring } \\
\text { pooled, women only }\end{array}$ & $\begin{array}{l}\text { Linear regression } \\
\text { I. crude } \\
\text { 2. multivariable adjusted for smoking, BMI, parity, generation } \\
\text { (measured at exams prior to menopause) }\end{array}$ \\
\hline \multirow{2}{*}{$\begin{array}{l}\text { Cognitive function } \\
\text { - MMSE at age } 65 \text { years (I. MMSE65X, } \\
\text { 2. MMSE65MVX) } \\
\text { - MMSE at the specified Offspring } \\
\text { exam (I. MMSE5X, 2. MMSE5MVX, I. } \\
\text { MMSE7X, 2. MMSE7MV, I. } \\
\text { MMSE5to7X, 2. MMSE5to7MVX) }\end{array}$} & 2 & $593(462)$ & $\begin{array}{l}\text { Cohort \& Offspring } \\
\text { pooled, exams at age } 65\end{array}$ & $\begin{array}{l}\text { Linear regression } \\
\text { Sex-specific } \\
\text { I. birth cohort } \\
\text { 2. multivariable adjusted for birth cohort, education, FSRP } \\
\text { measured at exam closest to age } 65 \text { years ( } 5 \text { year horizon) }\end{array}$ \\
\hline & 6 & $1038(913)$ & $\begin{array}{l}\text { Exam } 5 \\
\text { Exam } 7 \\
\text { Exam } 5 \text { \& } 7 \\
\text { average score }\end{array}$ & $\begin{array}{l}\text { Linear regression } \\
\text { Sex-specific } \\
\text { I. birth cohort } \\
\text { 2. multivariable adjusted for birth cohort, education, FSRP; } \\
\text { covariates measured at the specified exam }\end{array}$ \\
\hline $\begin{array}{l}\text { Physical Performance } \\
\text { - Hand grip (2. handgrip7x, } 2 . \\
\text { handgrips } 727 x) \\
\text { - Walking speed (2. walkingspeed7x, } 2 . \\
\text { walkingspeed727x) }\end{array}$ & 6 & 764 & $\begin{array}{l}\text { Exam } 7 \\
\text { Exam } 7 \text { and Exam } 27\end{array}$ & $\begin{array}{l}\text { Linear regression } \\
\text { Sex-specificf } \\
\text { I. age } \\
\text { 2. multivariable adjusted for age, height, weight at the } \\
\text { specified exam }\end{array}$ \\
\hline $\begin{array}{l}\text { Biologic Age by Osseographic } \\
\text { Scoring System (I. deltaOSSr, delta } \\
\text { OSSrf, deltaOSSrm) }\end{array}$ & 3 & 714 & $\begin{array}{l}\text { Offspring and Cohort } \\
\text { pooled } \\
\text { exam } 6 / 7 \text { and exam } 22\end{array}$ & $\begin{array}{l}\text { Linear regression } \\
\text { Sex- and cohort-specific ranked residuals§ } \\
\text { I. multivariable adjusted for age, height, BMI, menopause, } \\
\text { estrogen use }\end{array}$ \\
\hline
\end{tabular}

Residuals from these models were used as traits to test for association with SNP genotypes.

* The number preceding the variable name refers to the covariate adjustment in the last column of the table. The website with all results is found at http:// www.ncbi.nlm.nih.gov/projects/gap/cgi-bin/study.cgi?id=phs000007; ** MV = N for multivariable trait

$\neq$ cohort- and sex-specific residuals for traits that included both cohort and offspring; §cohort-specific for traits limited to one sex

$\mathrm{ALE}=$ average life expectancy, BMI = body mass index, Co-morbidity = cardiovascular disease and cancer, CVD = cardiovascular disease, FSRP = Framingham stroke risk profile, MMSE = mini-mental state exam, Risk factors = hypertension, diabetes, elevated cholesterol

on the $100 \mathrm{~K}$ Affymetrix GeneChip. There were 2036 SNPs in the LGV1 region on chromosome 4 previously linked to exceptional longevity [11]. Table 4 shows the candidate genes with SNPs associated with an FBAT or GEE p-value $<0.01$ for age at death including: FOXO1a, GAPDH, KL, LEPR, PON1, PSEN1, SOD2, and WRN and for morbidityfree survival at 65 years including:GHR, LEPR, MORF4L1, PON1, PTH, and WRN. Biologic age by OSS shared 2 SNPs in common with age at death: rs4943794 intronic to FOXO1 $a$ and rs911847 near SOD2.

\section{Discussion}

To our knowledge, this is the first dense GWAS of longevity and aging traits in a community-based sample of adults from two generations of the same families. Over 1300 men and women have detailed longevity and aging- 
Table 2: Aging and Longevity Phenotypest for FHS 100K Project: Results of Association and Linkage Analyses

2a. GEE, Top 5 p-values by Phenotype*

\begin{tabular}{|c|c|c|c|c|c|c|}
\hline Trait & SNP & Chromosome & Physical location & GEE p-value & FBAT p-value & Gene Region (within 60 kb) \\
\hline \multicolumn{7}{|c|}{ Age at death } \\
\hline & rsI528753 & 11 & $90,523,987$ & $8.1 \times 10^{-8}$ & 0.024 & \\
\hline & rs237I 208 & 7 & $81,982,510$ & $2.6 \times 10^{-6}$ & 0.031 & \\
\hline & rs 10496799 & 2 & $|39,26|, 40 \mid$ & $1.4 \times 10^{-5}$ & 0.735 & $\mathrm{NXPH} 2$ \\
\hline & rs 10489006 & 4 & $31,444,987$ & $3.6 \times 10^{-5}$ & 0.078 & \\
\hline & rs3757354 & 6 & $16,235,386$ & $6.4 \times 10^{-5}$ & 0.316 & MYLIP \\
\hline \multicolumn{7}{|c|}{ Morbidity-free survival at age 65} \\
\hline & rs|4|2337 & I & $165,350,299$ & $1.8 \times 10^{-9}$ & 0.505 & DPT \\
\hline & rs32566 & 5 & $5,845,507$ & $1.9 \times 10^{-9}$ & 0.323 & \\
\hline & rs 10484246 & 6 & $9,559,183$ & $8.4 \times 10^{-8}$ & 0.928 & \\
\hline & rs4831837 & 8 & $12,756,234$ & $4.7 \times 10^{-7}$ & 0.182 & \\
\hline & rs2639889 & 16 & $59,680,648$ & $9.4 \times 10^{-7}$ & 0.903 & \\
\hline \multicolumn{7}{|c|}{ Age at natural menopause $\ddagger$} \\
\hline & rs 10496265 & 2 & $81,580,466$ & $1.1 \times 10^{-8}$ & 0.001 & \\
\hline & rs 10496262* & 2 & $81,662,782$ & $3.3 \times 10^{-7}$ & 0.005 & \\
\hline & rs958672 & 2 & $154,896,075$ & $1.9 \times 10^{-6}$ & 0.087 & GALNTI3 \\
\hline & rs291353 & 1 & $232,046,939$ & $5.5 \times 10^{-6}$ & 0.035 & GNG4 \\
\hline & rs726336 & 5 & $163,911,906$ & $1.1 \times 10^{-5}$ & 0.125 & \\
\hline \multicolumn{7}{|c|}{ Walking speed exam 7} \\
\hline & rs7137869 & 12 & $118,452,366$ & $6.3 \times 10^{-7}$ & 0.009 & CCDC60 \\
\hline & rs7662116 & 4 & $154,375,569$ & $1.9 \times 10^{-5}$ & 0.016 & \\
\hline & rs7972859* & 12 & $118,452,765$ & $2.5 \times 10^{-5}$ & 0.005 & CCDC60 \\
\hline & rs9318312 & 13 & $74,489,506$ & $5.8 \times 10^{-5}$ & 0.266 & \\
\hline & rs1994854 & 4 & $78,124,824$ & $9.4 \times 10^{-5}$ & 0.280 & \\
\hline & rs7718104 & 5 & $122,183,258$ & $1.2 \times 10^{-4}$ & 0.011 & SNX2 \\
\hline \multicolumn{7}{|c|}{ Biologic age by osseographic scoring system } \\
\hline & rs|463605 & 12 & $30,005,150$ & $7.0 \times 10^{-8}$ & $5.3 \times 10^{-4}$ & \\
\hline & rs7176093 & 15 & $84,170,434$ & $7.4 \times 10^{-6}$ & 0.005 & $\underline{\text { KLHL25 }}$ \\
\hline & rs3772255 & 3 & $157,585,436$ & $8.2 \times 10^{-6}$ & 0.085 & KCNABI \\
\hline & rs726846 & 5 & $136,099,953$ & $1.1 \times 10^{-5}$ & 0.003 & \\
\hline & rs646983 & 13 & $29,413,553$ & $1.2 \times 10^{-5}$ & 0.003 & \\
\hline
\end{tabular}

2b. FBAT, Top 5 p-values by Phenotype*

\begin{tabular}{|c|c|c|c|c|c|c|}
\hline Trait & SNP & Chromosome & Physical location & GEE p-value & FBAT $p$-value & Gene Reg \\
\hline \multicolumn{7}{|c|}{ Age at death } \\
\hline & rs 10493513 & 1 & $73,091,610$ & 0.640 & $1.5 \times 10^{-6}$ & \\
\hline & rs10493514* & I & $73,092,533$ & 0.623 & $2.8 \times 10^{-6}$ & \\
\hline & rs668949l* & 1 & $73,064,050$ & 0.205 & $2.0 \times 10^{-5}$ & \\
\hline & rs 10493515 & I & $73,527,652$ & 0.225 & $2.3 \times 10^{-5}$ & \\
\hline & rs10493518* & I & $73,572,652$ & 0.191 & $3.6 \times 10^{-5}$ & \\
\hline & rs10493517* & I & $73,570,372$ & 0.215 & $4.2 \times 10^{-5}$ & \\
\hline & rs6657082* & 1 & $73,065,349$ & 0.224 & $5.5 \times 10^{-5}$ & \\
\hline & rs 10498263 & 14 & $19,285,288$ & 0.310 & $8.3 \times 10^{-5}$ & $\begin{array}{l}\text { OR4Q3 } \\
\text { OR4MI }\end{array}$ \\
\hline & rs191550I & 4 & $28,612,632$ & 0.383 & $1.1 \times 10^{-4}$ & \\
\hline & rsl40505I* & I & $73,060,505$ & 0.176 & $1.4 \times 10^{-4}$ & \\
\hline & rs6459623 & 6 & $|8,634,79|$ & 0.604 & $1.5 \times 10^{-4}$ & IBRDC2 \\
\hline
\end{tabular}


Table 2: Aging and Longevity Phenotypest for FHS 100K Project: Results of Association and Linkage Analyses (Continued)

\begin{tabular}{|c|c|c|c|c|c|}
\hline \multicolumn{6}{|c|}{ Morbidity-free survival at age 65} \\
\hline rs 10509200 & 10 & $65,296,567$ & 0.613 & $7.0 \times 10^{-5}$ & \\
\hline rs965036 & 6 & $20,099,022$ & 0.550 & $8.6 \times 10^{-5}$ & \\
\hline rs720565 & 6 & $136,834,657$ & 0.014 & $9.8 \times 10^{-5}$ & MAP7 \\
\hline rsII92372 & 2 & $84,923,204$ & 0.094 & $9.9 \times 10^{-5}$ & \\
\hline rs 10505239 & 8 & $115,976,403$ & 0.141 & $1.3 \times 10^{-4}$ & \\
\hline \multicolumn{6}{|c|}{ Age at natural menopause $\dagger$} \\
\hline rs959702 & 10 & $2,139,260$ & 0.003 & $1.4 \times 10^{-5}$ & \\
\hline rs7165378 & 15 & $69,478,558$ & 0.006 & $6.4 \times 10^{-5}$ & \\
\hline rs997161 & 10 & $130,876,127$ & 0.074 & $8.6 \times 10^{-5}$ & \\
\hline rsl65284 & I & $91,235,574$ & 0.006 & $8.6 \times 10^{-5}$ & ZNF644 \\
\hline rs2280585 & 3 & $64,882,324$ & 0.884 & $1.1 \times 10^{-4}$ & \\
\hline \multicolumn{6}{|c|}{ Walking speed exam 7} \\
\hline rs447I448 & 11 & $86,760,972$ & 0.175 & $3.8 \times 10^{-6}$ & TMEMI35 \\
\hline rs336963 & 5 & $83,005,460$ & 0.570 & $2.6 \times 10^{-5}$ & HAPLNI \\
\hline rs7862683 & 9 & $18,257,947$ & 0.001 & $4.9 \times 10^{-5}$ & \\
\hline rs9317757 & 13 & $68,458,430$ & 0.252 & $7.8 \times 10^{-5}$ & \\
\hline rs10501636* & 11 & $86,789,967$ & 0.355 & $1.1 \times 10^{-4}$ & \\
\hline rs2340392 & 3 & $80,440,990$ & 0.002 & $1.7 \times 10^{-4}$ & \\
\hline \multicolumn{6}{|c|}{ Biologic age by osseographic scoring system } \\
\hline rs/380703 & 2 & $57,852,938$ & 0.008 & $1.1 \times 10^{-5}$ & \\
\hline rs324702 & 4 & $77,094,969$ & 0.390 & $3.3 \times 10^{-5}$ & PPEF2 \\
\hline rs324735 & 4 & $77,062,193$ & 0.096 & $7.6 \times 10^{-5}$ & \\
\hline rsII06184 & 2 & $10,914,125$ & 0.006 & $8.8 \times 10^{-5}$ & PDIA6 \\
\hline rs604578 & 18 & $30,923,438$ & 0.004 & $9.5 \times 10^{-5}$ & MAPRE2 \\
\hline
\end{tabular}

2c Linkagell Peaks with LOD scores $\geq 2.0$

\begin{tabular}{|c|c|c|c|c|c|c|}
\hline Trait & $\begin{array}{l}\text { SNP closest to } \\
\text { linkage peak }\end{array}$ & Chromosome & Physical location & $\begin{array}{r}\text { I.5 - LOD } \\
\text { support } \\
\text { interval start }\end{array}$ & $\begin{array}{r}\text { I.5 - LOD } \\
\text { support } \\
\text { interval end }\end{array}$ & LOD score \\
\hline
\end{tabular}

Age at natural menopause

\begin{tabular}{|c|c|c|c|c|c|}
\hline rs 1371217 & 4 & $182,890,808$ & $|78,67|, 796$ & $186,905,362$ & 2.08 \\
\hline rs 10509024 & 10 & $56,567,832$ & $36,084,470$ & $70,573,011$ & 2.39 \\
\hline rs47935I3 & 17 & $66,429,892$ & $60,635,492$ & $69,5 \mid 2,021$ & 2.48 \\
\hline \multicolumn{6}{|c|}{ ng speed, exam 7} \\
\hline rs276926I & I & II 3,278,949 & $107,080,550$ & $144,332,709$ & 2.30 \\
\hline rs921055 & 2 & $233,522,842$ & $229,328,008$ & $242,14 \mid, 304$ & 2.13 \\
\hline rs2602044 & 3 & $109,427,883$ & $102,255,525$ & $111,604,019$ & 3.38 \\
\hline rs80III73 & 14 & $96,927,485$ & $96,342,135$ & $100,389,787$ & 2.69 \\
\hline rs|362626 & 16 & $4,489,227$ & 205,160 & $10,344,522$ & 2.05 \\
\hline \multicolumn{6}{|c|}{ jic age by osseographic scoring system } \\
\hline rs353810 & 9 & $86,258,745$ & $8 I, 44 I, 976$ & $92,220,168$ & 3.26 \\
\hline rs I20398| & 16 & 205,160 & 205,160 & $7,431,239$ & 2.49 \\
\hline rs2248383 & 21 & $35,|5|, 81 \mid$ & $27,4|2,7| 6$ & $40,940,879$ & 2.22 \\
\hline
\end{tabular}

SNP criteria: Autosomal SNPs with genotypic call rate $\geq 80 \%$, minor allele frequency $\geq 10 \%$, Hardy-Weinberg test $p>0.001$, and $\geq 10$ informative families for FBAT

* For each phenotype SNPs are ranked by p-value. A SNP in LD $\left(r^{2}>0.8\right)$ with a higher ranked SNP, is identified with an asterisk. All SNPs for a phenotype are listed until 5 independent SNPs are identified. Thus, for some phenotypes more than 5 SNPs are listed. For the age at death trait, the FBAT analysis identified two areas on chromosome I in LD, with $r^{2}=.5-.6$ between the two regions and $r^{2}$ of nearly 1.0 within the region.

† Multivariable-adjusted trait results are presented

$\ddagger$ Trait had $<500$ participants in the sample.

TResults limited to traits presented 
Table 3: All Significant SNP Associations (GEE or FBAT p-value $<0.01$ ) for at least Two Traits

\begin{tabular}{|c|c|c|c|c|c|c|c|c|c|}
\hline \multicolumn{10}{|c|}{ 3a. FBAT: Age at Death and Morbidity-Free Survival at 65 years } \\
\hline Trait I & Trait 2 & SNP & Chr & $\begin{array}{l}\text { Physical } \\
\text { Position }\end{array}$ & Gene & $\begin{array}{l}\text { Trait I GEE } \\
\text { p-value }\end{array}$ & $\begin{array}{l}\text { Trait I FBAT } \\
\text { p-value }\end{array}$ & $\begin{array}{l}\text { Trait } 2 \text { GEE } \\
\text { p-value }\end{array}$ & $\begin{array}{c}\text { Trait } 2 \text { FBAT } \\
\text { p-value }\end{array}$ \\
\hline \multirow[t]{7}{*}{$\begin{array}{l}\text { Age at } \\
\text { Death }\end{array}$} & $\begin{array}{l}\text { Morbidity- } \\
\text { free at } 65\end{array}$ & rs6682403 & 1 & $234,743,324$ & & 0.849 & 0.004 & 0.106 & 0.004 \\
\hline & & rs10488907 & 4 & $113,669,709$ & ALPKI & 0.452 & 0.008 & 0.336 & 0.009 \\
\hline & & rs 17190837 & 9 & $|3,39|, 548$ & & 0.010 & 0.009 & 0.097 & 0.004 \\
\hline & & rs4752977 & 11 & $47,257,005$ & MADD & 0.736 & 0.008 & 0.002 & 0.009 \\
\hline & & rsl0506274 & 12 & $80,103,932$ & & 0.531 & 0.001 & 0.989 & 0.001 \\
\hline & & rs283II54 & 21 & $28,059,331$ & & 0.323 & 0.008 & 0.358 & 0.006 \\
\hline & & rs $243725^{*}$ & 21 & $28,060,803$ & & 0.264 & 0.007 & 0.359 & 0.008 \\
\hline
\end{tabular}

$*_{r}^{2}>0.80$ with the preceding SNP

\begin{tabular}{|c|c|c|c|c|c|c|c|c|c|}
\hline \multicolumn{10}{|c|}{ 3b. GEE: Age at Death and Morbidity-Free Survival at 65 years } \\
\hline Trait I & Trait 2 & SNP & Chr & $\begin{array}{l}\text { Physical } \\
\text { Position }\end{array}$ & Gene & $\begin{array}{c}\text { Trait I GEE } \\
\text { p-value }\end{array}$ & $\begin{array}{c}\text { Trait I FBAT } \\
\text { p-value }\end{array}$ & $\begin{array}{c}\text { Trait } 2 \text { GEE } \\
\text { p-value }\end{array}$ & $\begin{array}{c}\text { Trait } 2 \text { FBAT } \\
\text { p-value }\end{array}$ \\
\hline \multirow[t]{9}{*}{$\begin{array}{l}\text { Age at } \\
\text { Death }\end{array}$} & $\begin{array}{l}\text { Morbidity- } \\
\text { free at } 65\end{array}$ & rs930826I & 1 & $113,603,160$ & MAGI3 & 0.009 & 0.156 & 0.002 & 0.900 \\
\hline & & rs 10490518 & 2 & $31,223,004$ & GALNTI4 & 0.009 & 0.373 & 0.010 & 0.948 \\
\hline & & rs2374983 & 7 & $94,516,375$ & $\begin{array}{l}\text { PPPIR9A/ } \\
\text { PONI }\end{array}$ & 0.006 & 0.980 & 0.007 & 0.727 \\
\hline & & rs655883 & 11 & $98,994,584$ & CNTN5 & 0.006 & 0.390 & 0.001 & 0.293 \\
\hline & & rs|368850 & 11 & $130,433,518$ & & 0.004 & 0.387 & 0.005 & 0.292 \\
\hline & & rs4943II6 & 13 & $32,995,650$ & STARDI 3 & 0.006 & 0.205 & 0.008 & 0.049 \\
\hline & & rs2254191 & 13 & $45,344,403$ & & 0.007 & 0.425 & 0.004 & 0.116 \\
\hline & & rsl620210 & 13 & $45,759,488$ & $\mathrm{Cl} 3$ orf 18 & 0.001 & 0.161 & 0.004 & 0.068 \\
\hline & & rs2823322 & 21 & $|5,8| 4,903$ & & 0.0004 & 0.045 & 0.006 & 0.029 \\
\hline
\end{tabular}

3c. FBAT: Biologic Age and Walking Speed

\begin{tabular}{|c|c|c|c|c|c|c|c|c|c|}
\hline Trait I & Trait 2 & SNP & Chr & $\begin{array}{l}\text { Physical } \\
\text { Position }\end{array}$ & Gene & $\begin{array}{c}\text { Trait I GEE } \\
\text { p-value }\end{array}$ & $\begin{array}{c}\text { Trait I FBAT } \\
\text { p-value }\end{array}$ & $\begin{array}{c}\text { Trait } 2 \text { GEE } \\
\text { p-value }\end{array}$ & $\begin{array}{c}\text { Trait } 2 \text { FBAT } \\
\text { p-value }\end{array}$ \\
\hline Biologic age & Walking speed & rs873348 & 4 & $178,246,509$ & & 0.135 & 0.004 & 0.114 & 0.006 \\
\hline Biologic age & Walking speed & rs|052036I* & 4 & $178,247,037$ & & 0.074 & 0.006 & 0.054 & 0.005 \\
\hline Biologic age & Walking speed & rs31564 & 5 & $135,258,152$ & IL9 & 0.015 & 0.001 & 0.008 & 0.002 \\
\hline Biologic age & Walking speed & rs 1862345 & 5 & $148,018,498$ & HTR4 & 0.172 & 0.0002 & 0.399 & 0.008 \\
\hline Biologic age & Walking speed & rs7844834 & 8 & $11,323,556$ & $\begin{array}{c}\text { C8orf } 12 \mid \mathrm{C} 8 \text { or } \\
\mathrm{f} / 3\end{array}$ & 0.017 & 0.004 & 0.011 & 0.004 \\
\hline Biologic age & Walking speed & rs952658 & 12 & $20,756,568$ & SLCOICI & 0.024 & 0.008 & 0.935 & 0.006 \\
\hline Biologic age & Walking speed & rs6487366 & 12 & $23,994,617$ & sox5 & 0.017 & 0.003 & 0.959 & 0.004 \\
\hline Biologic age & Walking speed & rs7135493 & 12 & $28,134,847$ & & 0.020 & 0.006 & 0.033 & 0.004 \\
\hline Biologic age & Walking speed & rsl0492036 & 12 & I $24,728,934$ & & 0.308 & 0.005 & 0.169 & 0.009 \\
\hline Biologic age & Walking speed & rs1978945 & 13 & $105,64 \mid, 257$ & & 0.093 & 0.009 & 0.031 & 0.006 \\
\hline Biologic age & Walking speed & rs2165723* & 13 & $105,641,610$ & & 0.046 & 0.010 & 0.027 & 0.003 \\
\hline Biologic age & Walking speed & rsl049265I* & 13 & $105,64 \mid, 634$ & & 0.083 & 0.009 & 0.023 & 0.001 \\
\hline Biologic age & Walking speed & rs9301।I2* & 13 & $105,642,018$ & & 0.097 & 0.004 & 0.053 & 0.003 \\
\hline
\end{tabular}

$* r^{2}>0.8$ with the preceding SNP (calculated if the distance is $<250,000$ base pairs)

3d. GEE: Biologic Age and Walking Speed

\begin{tabular}{|c|c|c|c|c|c|c|c|c|c|}
\hline Trait I & Trait 2 & SNP & Chr & $\begin{array}{l}\text { Physical } \\
\text { Position }\end{array}$ & Gene & $\begin{array}{l}\text { Trait I GEE } \\
\text { p-value }\end{array}$ & $\begin{array}{c}\text { Trait I FBAT } \\
\text { p-value }\end{array}$ & $\begin{array}{c}\text { Trait } 2 \text { GEE } \\
\text { p-value }\end{array}$ & $\begin{array}{c}\text { Trait } 2 \text { FBAT } \\
\text { p-value }\end{array}$ \\
\hline Biologic Age & Walking speed & rs|474827 & 6 & $|34,886,0| \mid$ & & 0.007 & 0.746 & 0.000 & 0.233 \\
\hline Biologic Age & Walking speed & rsl023I64I & 7 & $119,166,342$ & & 0.004 & 0.077 & 0.009 & 0.278 \\
\hline Biologic Age & Walking speed & rs310575 & 8 & $51,603,257$ & SNTGI & 0.008 & 0.209 & 0.003 & 0.007 \\
\hline Biologic Age & Walking speed & rsl0520603 & 15 & $84,170,955$ & & 0.009 & 0.008 & 0.003 & 0.050 \\
\hline Biologic Age & Walking speed & rs7166323* & 15 & $84,171,745$ & & 0.009 & 0.012 & 0.003 & 0.065 \\
\hline Biologic Age & Walking speed & rs2215921 & 16 & $9,604,834$ & & 0.007 & 0.049 & 0.001 & 0.316 \\
\hline
\end{tabular}

$* r^{2}>0.8$ with the preceding SNP (calculated if the distance is $<250,000$ base pairs) 
Table 4: All Significant SNP Associations with Selected Longevity Candidate Genes* (FBAT or GEE p-value $<0.0$ I)

\begin{tabular}{|c|c|c|c|c|c|c|c|c|}
\hline Trait & Gene & SNP & Chr & $\begin{array}{l}\text { Physical } \\
\text { Position }\end{array}$ & FBAT p-value & GEE p-value & SNP function & $\begin{array}{l}\text { SNP position relative } \\
\text { to gene (up to } 60 \mathrm{~kb} \text { ) }\end{array}$ \\
\hline \multirow[t]{14}{*}{ Age at death } & FOXOla & rs4943794 & 13 & $40,071,408$ & 0.068 & 0.00028 & Intron & in \\
\hline & & rs 10507486 & 13 & $40,084,501$ & 0.043 & 0.00013 & Intron & in \\
\hline & GAPDH $\dagger$ & rs4764600 & 12 & $6,472,241$ & 0.833 & 0.005 & Locus/intron & near \\
\hline & KL & rs683907 & 13 & $32,522,175$ & 0.009 & 0.507 & Intron & in \\
\hline & & rs687045 & 13 & $32,522,889$ & 0.007 & 0.712 & Intron & in \\
\hline & LEPR & rs|475398 & 1 & $65,695,278$ & 0.069 & 0.005 & Untranslated & in \\
\hline & & rs I34398| & 1 & $65,757,349$ & 0.031 & 0.006 & Intron & in \\
\hline & & rs 10493379 & 1 & $65,757,948$ & 0.015 & 0.004 & Intron & in \\
\hline & & rs 2154380 & 1 & $65,769,462$ & 0.004 & 0.003 & Intron & in \\
\hline & & rs6669117 & 1 & $65,773,093$ & 0.050 & 0.007 & Intron & in \\
\hline & PONI & rs2374983 & 7 & $94,516,375$ & 0.980 & 0.006 & Intron & near \\
\hline & PSEN I & rs 362356 & 14 & $72,708,382$ & 0.005 & 0.130 & Intron & in \\
\hline & SOD2 & rs91 I 847 & 6 & $160,039,379$ & 0.358 & 0.005 & Unknown & near \\
\hline & WRN $\ddagger$ & rs 2543600 & 8 & $30,969,282$ & 0.182 & $4.2 \times 10^{-6}$ & Unknown & near \\
\hline \multirow[t]{8}{*}{ Morbidity-free survival at age 65} & GHR & rs719756 & 5 & $42,761,386$ & 0.003 & 0.676 & Unknown & near \\
\hline & LEPR & rs II7I 278 & 1 & $65,700,167$ & 0.042 & 0.003 & Untranslated & in \\
\hline & & rs3790426 & 1 & $65,755,040$ & 0.460 & 0.002 & Intron & in \\
\hline & MORF4LI & rs|383636 & 15 & $76,893,275$ & 0.458 & 0.007 & Unknown & near \\
\hline & PONI & rs2374983 & 7 & $94,516,375$ & 0.727 & 0.007 & Intron & near \\
\hline & & rs854523 & 7 & $94,542,884$ & 0.850 & 0.007 & Intron & in \\
\hline & PTH & rs 10500784 & $\mathrm{II}$ & $13,530,40 \mid$ & 0.010 & 0.990 & Unknown & near \\
\hline & WRN $\ddagger$ & rs 2725369 & 8 & $30,970,566$ & 0.113 & 0.003 & Unknown & near \\
\hline \multirow[t]{6}{*}{ Biologic Age by OSS } & Foxola & rs 1923249 & 13 & $40,041,881$ & 0.006 & 0.004 & Intron & in \\
\hline & & rs4943794 & 13 & $40,07 \mid, 408$ & 0.009 & 0.016 & Intron & in \\
\hline & HSPA9 & rs 256014 & 5 & $137,930,983$ & 0.101 & 0.005 & Intron & in \\
\hline & LASS6 & rs1002666 & 2 & $169,303,525$ & 0.001 & 0.008 & Intron & in \\
\hline & SOD2 & rs91 I 847 & 6 & $160,039,379$ & 0.024 & 0.009 & Unknown & near \\
\hline & TLR4 & rs1927914 & 9 & $117,544,279$ & 0.007 & 0.401 & Locus & near \\
\hline \multirow[t]{7}{*}{ Walking speed } & ESRI & rs932236I & 6 & $|52,55|, 257$ & 0.124 & 0.0089 & Intron & in \\
\hline & LASS6 & rs6433083 & 2 & $|69,324,82|$ & 0.232 & 0.006 & Intron & in \\
\hline & NR3CI & rs 2918418 & 5 & $142,703,566$ & 0.005 & 0.081 & Intron & in \\
\hline & & rs 10515522 & 5 & $142,738,587$ & 0.004 & 0.084 & Intron & in \\
\hline & SODI & rs 2833485 & 21 & $32,000,796$ & 0.008 & 0.507 & Locus/intron & in \\
\hline & TERF2 & rs728546 & 16 & $68,013,029$ & 0.0045 & 0.533 & Unknown & near \\
\hline & FASLG & rs6700734 & 1 & $169,362,468$ & 0.003 & 0.029 & Intron & in \\
\hline
\end{tabular}

*79 genes identified from NCBI, SAGE ke, and GenAge databases; 12 genes with
chip, results for this region available on the web
†The most strongly associated SNP near GAPDH is actually closer to MRPL5 I
†The most strongly associated SNP near WRN is actually closer to PURG

related phenotypes and 100K SNP genotyping results available on the web. This resource has the potential to detect novel susceptibility genes for human longevity and aging and to examine the relevance of promising candidate gene associations reported in animal models to human aging. We describe several strategies to prioritize SNP associations in this unique resource to enhance the discovery of various genes and pathways that contribute to the control of human longevity. Furthermore, FHS investigators are part of the NIA sponsored Longevity Consortium http://www.longevityconsortium.org which offers the opportunity of collaboration with other investigators to replicate important findings in additional cohorts.

In our untargeted approach of ranking SNP associations by the strength of the p-value, 2 intronic FOXO1a SNPs were associated with age at death. One of these SNPs (rs4943794) also was associated with biologic age by OSS in our a priori evaluation of select candidate genes. FOXO (forkhead box group O) transcription factors are targets of insulin-like signaling and are involved in a diverse set of physiological functions including DNA repair and resistance to oxidative stress $[33,34]$. Further, FOXO plays a role in lifespan extension in C. elegans and Drosophila [35]. Studies of this gene in humans are limited; two case-control studies have not identified an association between FOXO1 $a$ and longevity [36,37]. However, the prospective population-based Leiden 85-plus Study found that FOXO1a was associated with increased mortality attributable to diabetes related deaths in participants aged 85 years and older [38]. The Leiden 85-plus Study also reported that genetic variation causing a reduction in insulin/IGF-1 signaling resulted in improved old age survival among women [20]. However, that report examined other genes in the insulin/insulin-like signaling pathway and did not specifically examine FOXO1a. Finally, the untargeted approach to SNP selection also identified a SNP near FOXO3a associated with age at natural menopause. This gene has been implicated in oocyte death, 
depletion of functioning ovarian follicles, and infertility in mice $[39,40]$ and thus represents a plausible candidate gene for menopause. Most positive common gene variantdisease association studies have failed replication [41] including reports on exceptional longevity. Haplotypebased fine mapping of the region on chromosome 4 linked to human longevity initially suggested the MTP gene, a gene important in lipoprotein synthesis, was associated with longevity [21]. However, this association failed replication in a French cohort of long-lived individuals and subsequent case-control studies of nonagenarians $[22,42]$. Beekman, et al [43] found neither linkage to chromosome 4 nor association with the MTP gene and longevity among nonagenarians in the Leiden Longevity Study. Meta-analyses implicated admixture of the control sample in the original report as an explanation for the presumed false-positive association. Thus, our findings are hypothesis generating and their importance can not be determined without evidence of consistent replication in other populations.

We examined pleiotropic effects by identifying SNP associations across two pairs of related traits. One SNP near PON1 emerged as associated with both age at death and morbidity-free survival. Surprisingly, there were relatively few SNPs associated with both traits; prior work had suggested that longevity per se and healthy aging may share common genetic pathways $[11,12]$. However, morbidityfree survival was measured at age 65 years, it is possible that as our participants age morbidity-free survival defined at age 75 or 85 years will share additional SNP associations with our longevity trait, age at death. A SNP near SOX5, a gene potentially related to musculoskeletal function was associated with both biologic age by OSS and walking speed.

Our strategy of selecting SNPs in candidate genes and regions previously reported to be associated with longevity yielded interesting findings. For age at death, we identified SNPs in or near several genes including $K L$, $L E P R$, PON1, SOD2, and WRN. Defects in the WRN gene are the cause of Werner Syndrome, an autosomal recessive disorder characterized by premature aging. A longitudinal study of ageing Danish twins recently reported a possible association between a successful aging trait and 3 SNPs in the WRN gene [44]. We were unable to determine if our SNP (rs2725369) was in LD with the SNPs in the prior report because the SNPs were not included in HapMap. Mutations in the KL (Klotho) gene in the mouse lead to a syndrome resembling human aging [45-47]. There has been one report linking a functional variant of the $K L$ gene to human longevity [15]. Thus, results from this GWAS may direct resources to the most relevant candidate genes and pathways for further investigation in humans.
Several important limitations merit comment. First, we acknowledge that there may be a survival bias as participants in this sample had to survive to provide DNA (first systematic DNA collection began 1995) and hence are likely healthier than the full FHS sample. To ameliorate this issue, we adjusted for covariates using the full Framingham sample, and used the residual traits for the subset of individuals genotyped using the 100K Affymetrix GeneChip to test for association with the SNPs using linear regression models. Residual traits from Cox and logistic models typically are not ideally distributed for linear regression models, but our adjustment method using the full sample precludes the testing of SNP associations with age at death and morbidity-free survival using Cox and logistic models. Second, the 100K Affymetrix GeneChip provides limited coverage of the genome; many of our a priori candidate genes did not have any SNP coverage on the chip. For example, several genes that have been studied in model organisms or even in humans such as $A C E$, Lamin A, SIRT2 and SIRT3, had no SNPs within $60 \mathrm{~kb}$ of the gene on the $100 \mathrm{~K}$ Affymetrix GeneChip. However, genotyping is near-complete for the NHLBI funded $550 \mathrm{~K}$ genome-wide scan on all FHS participants. This will enable deeper exploration of our initial 100K SNP associations in a larger sample with denser coverage of the genome. Third, in this analysis we did not examine epistasis or gene-environment interactions which may modify the associations in this study. Importantly, this study is hypothesis generating. Our findings need to be replicated in other samples.

\section{Conclusion}

In summary, the untargeted genome-wide approach to detect genetic associations with longevity and aging traits provides an opportunity to identify novel biologic pathways related to lifespan control. GWAS also have the potential to direct investigators of human aging to the most promising candidate gene associations and biologic pathways reported to regulate lifespan in animal models. Enhancing our understanding of the mechanisms responsible for aging may in turn identify directions for health promotion and disease prevention efforts in middle-aged and older adults so that older persons can enjoy more time in good health. These data generate hypotheses regarding novel biologic pathways contributing to longevity and healthy aging and serve as a resource for replication of findings from other population-based samples.

\section{Abbreviations}

$\mathrm{CVD}=$ cardiovascular disease $; \mathrm{FBAT}=$ family-based association test; FHS = Framingham Heart Study; GEE = generalized estimating equations; GWAS = genome-wide association study; $\mathrm{LD}=$ linkage disequilibrium; $\mathrm{LOD}=$ logarithm of the odds; NCBI = National Center Biotechnology Information; OSS = osseographic scoring system; 
$\mathrm{SNP}=$ single nucleotide polymorphism; TIA $=$ transient ischemic attack.

\section{Competing interests}

The authors declare that they have no competing interests.

\section{Authors' contributions}

All authors have made substantial contributions to conception and design or acquisition of phenotypic data. JMM, KL, EJB, CG, DK, DPK, JMM, MJP, RBD contributed to the analysis and interpretation of data. JMM, KL, EJB, DK, DPK, SS have been involved in drafting the manuscript or revising it critically for important intellectual content. All authors read and approved the final manuscript.

\section{Additional material}

\section{Additional file 1}

Candidate Gene List for FHS $100 \mathrm{~K}$ Longevity and Aging Traits

Click here for file

[http://www.biomedcentral.com/content/supplementary/1471-

2350-8-S1-S13-S1.doc]

\section{Acknowledgements}

NHLBI's Framingham Heart Study is supported by contract number NOIHC-25 I95. This work was also supported in part by ROI AR/AG 4I398 and AG02832I.

FHS I00K analyses were conducted using the Boston University.

Linux Cluster for Genetic Analysis (LinGA) funded by the NIH NCRR (National Center for Research Resources) Shared Instrumentation grant ISIORRI63736-0IAI http://www.bu.edu/dbin/sph/departments/biostatis tics/linga publications.php.

We thank the FHS participants for their ongoing participation and dedication to the study making this work possible.

This article has been published as part of BMC Medical Genetics Volume 8 Supplement I, 2007: The Framingham Heart Study 100,000 single nucleotide polymorphisms resource. The full contents of the supplement are available online at http://www.biomedcentral.com//47I-2350/8? issue=SI.

\section{References}

I. Herskind AM, McGue M, Holm NV, Sorensen TI, Harvald B, Vaupel JW: The heritability of human longevity: a population-based study of 2872 Danish twin pairs born 1870-1900. HumGenet 1996, 97(3):319.

2. lachine IA, Holm NV, Harris JR, Begun AZ, lachina MK, Laitinen M, Kaprio J, Yashin Al: How heritable is individual susceptibility to death? The results of an analysis of survival data on Danish, Swedish and Finnish twins. TwinRes 1998, I(4): 196.

3. Ljungquist B, Berg S, Lanke J, McClearn GE, Pedersen NL: The effect of genetic factors for longevity: a comparison of identical and fraternal twins in the Swedish Twin Registry. JGerontolA BiolSciMedSci 1998, 53(6):M44I.

4. McGue M, Vaupel JW, Holm N, Harvald $B$ : Longevity is moderately heritable in a sample of Danish twins born 1870-1880. JGerontol 1993, 48(6):B237.
5. Hijelmborg JV, lachine I, Skytthe A, Vaupel JW, McGue M, Koskenvuo M, Kaprio J, Pedersen NL, Christensen K: Genetic influence on human lifespan and longevity. HumGenet 2006, I I 9(3):3 I2.

6. Reed T, Dick DM: Heritability and validity of healthy physical aging (wellness) in elderly male twins. TwinRes 2003, 6(3):227.

7. Carmelli D, Kelly-Hayes M, Wolf PA, Swan GE, Jack LM, Reed T, Guralnik JM: The contribution of genetic influences to measures of lower-extremity function in older male twins. JGerontolA BiolSciMedSci 2000, 55(I):B49.

8. Reed T, Fabsitz RR, Selby JV, Carmelli D: Genetic influences and grip strength norms in the NHLBI twin study males aged 5969. AnnHumBiol I99I, I 8(5):425.

9. Swan GE, Carmelli D, Reed T, Harshfield GA, Fabsitz RR, Eslinger PJ: Heritability of cognitive performance in aging twins. The National Heart, Lung, and Blood Institute Twin Study. ArchNeurol 1990, 47(3):259.

10. Karasik D, Hannan MT, Cupples LA, Felson DT, Kiel DP: Genetic contribution to biological aging: the Framingham Study. JGerontolA BiolSciMedSci 2004, 59(3):2 I8.

II. Puca AA, Daly MJ, Brewster SJ, Matise TC, Barrett J, Shea-Drinkwater M, Kang S, Joyce E, Nicoli J, Benson E, Kunkel LM, Perls T: A genome-wide scan for linkage to human exceptional longevity identifies a locus on chromosome 4. ProcNat/AcadSciUSA 200I, 98( ( 8): 10505

12. Reed T, Dick DM, Uniacke SK, Foroud T, Nichols WC: Genomewide scan for a healthy aging phenotype provides support for a locus near D4SI564 promoting healthy aging. JGerontolA BiolSciMedSci 2004, 59(3):227.

13. Cheng CL, Gao TQ, Wang Z, Li DD: Role of insulin/insulin-like growth factor I signaling pathway in longevity. World JGastroenterol 2005, I I ( I3): I89I.

14. Katic M, Kahn CR: The role of insulin and IGF-I signaling in longevity. Cell MolLife Sci 2005, 62(3):320.

15. Arking DE, Atzmon G, Arking A, Barzilai N, Dietz HC: Association between a functional variant of the KLOTHO gene and highdensity lipoprotein cholesterol, blood pressure, stroke, and longevity. CircRes 2005, 96(4):4I2.

16. Bellizzi D, Rose G, Cavalcante P, Covello G, Dato S, De Rango F, Greco V, Maggiolini M, Feraco E, Mari V, Franceschi C, Passarino G De Benedictis G: A novel VNTR enhancer within the SIRT3 gene, a human homologue of SIR2, is associated with survival at oldest ages. Genomics 2005, 85(2):258.

17. Browner WS, Kahn AJ, Ziv E, Reiner AP, Oshima J, Cawthon RM, Hsueh WC, Cummings SR: The genetics of human longevity. AmJMed 2004, I I 7(I I):85I.

18. Hadley EC, Rossi WK: Exceptional survival in human populations: National Institute on Aging perspectives and programs. MechAgeing Dev 2005, I 26(2):23I.

19. Rose G, Dato S, Altomare K, Bellizzi D, Garasto S, Greco V, Passarino G, Feraco E, Mari V, Barbi C, Bonafe M, Franceschi C, Tan Q, Boiko S, Yashin Al, De Benedictis G: Variability of the SIRT3 gene, human silent information regulator Sir2 homologue, and survivorship in the elderly. ExpGerontol 2003, 38(10): 1065.

20. van Heemst D, Beekman M, Mooijaart SP, Heijmans BT, Brandt BW, Zwaan BJ, Slagboom PE, Westendorp RG: Reduced insulin/IGF-I signalling and human longevity. Aging Cell 2005, 4(2):79.

21. Geesaman BJ, Benson E, Brewster SJ, Kunkel LM, Blanche H, Thomas G, Perls TT, Daly MJ, Puca AA: Haplotype-based identification of a microsomal transfer protein marker associated with the human lifespan. ProcNatiAcadSciUSA 2003, 100(24): 14115.

22. Nebel A, Croucher PJ, Stiegeler R, Nikolaus S, Krawczak M, Schreiber $\mathrm{S}$ : No association between microsomal triglyceride transfer protein (MTP) haplotype and longevity in humans. ProcNatIAcadSciUSA 2005, I 02(22):7906.

23. Hirschhorn JN, Daly MJ: Genome-wide association studies for common diseases and complex traits. NatRevGenet 2005, 6(2):95.

24. Cupples LA, Arruda H, Benjamin EJ, D'Agostino RB Sr, Demissie S, DeStefano AL, Dupuis J, Falls K, Fox CS, Gottlieb DJ, Govindaraju DR, Guo CY, Heard-Costa N, Hwang SJ, Kathiresan S, Kiel DP, Laramie JM, Larson MG, Levy D, Liu CY, Lunetta KL, Mailman MD, Manning AK, Meigs JB, Murabito JM, Newton-Cheh C, O'Connor GT, O'Donnell CJ, Pandey MA, Seshadri S, Vasan RS, Wang ZY, Wilk JB, Wolf PA, Yang Q, Atwood LD: The Framingham Heart Study I00K SNP genome-wide association study resource: Overview of 
17 phenotype working group reports. BMC Med Genet 2007 , 8(SuppI I):SI.

25. Survival following initial cardiovascular disease events: $\mathbf{3 0}$ year follow-up. In The Framingham Heart Study: An epidemiological investigation of cardiovascular disease Edited by: Kannel WB, Wolf PA, Garrison RJ. Bethseda, MD: NHLBI, NIH; 1988.

26. Murabito JM, Yang Q, Fox C, Wilson PW, Cupples LA: Heritability of age at natural menopause in the Framingham Heart Study. J Clin Endocrinol Metab 2005, 90(6):3427-3430.

27. Murabito JM, Yang Q, Fox CS, Cupples LA: Genome-wide linkage analysis to age at natural menopause in a community-based sample: the Framingham Heart Study. Fertil Steril 2005 84(6): 1674-1679.

28. SAS/STAT software, Version 8.2. SAS System for Windows. Copyright 1999-200I. SAS Institute Inc. Cary, NC, USA; 1999.

29. Karasik D, Demissie S, Cupples LA, Kiel DP: Disentangling the genetic determinants of human aging: biological age as an alternative to the use of survival measures. JGerontolA BiolSciMedSci 2005, 60(5):574.

30. 2006 [http://sageke.sciencemag.org/cgi/genesdb].

31. Karolchik D, Baertsch R, Diekhans M, Furey TS, Hinrichs A, Lu YT, Roskin KM, Schwartz M, Sugnet CW, Thomas DJ, Weber RJ, Haussler D, Kent W]: The UCSC Genome Browser Database. Nucleic Acids Res 2003, 3 I (I):5I.

32. Kent WJ, Sugnet CW, Furey TS, Roskin KM, Pringle TH, Zahler AM, Haussler D: The human genome browser at UCSC. Genome Res 2002, I 2(6): 996.

33. Barthel A, Schmoll D, Unterman TG: FoxO proteins in insulin action and metabolism. Trends EndocrinolMetab 2005, 16(4): 183.

34. Lam EW, Francis RE, Petkovic M: FOXO transcription factors: key regulators of cell fate. BiochemSoc Trans 2006, 34(Pt 5):722

35. Hwangbo DS, Gershman B, Tu MP, Palmer M, Tatar M: Drosophila dFOXO controls lifespan and regulates insulin signalling in brain and fat body. Nature 2004, 429(699I):562.

36. Bonafe M, Barbieri M, Marchegiani F, Olivieri F, Ragno E, Giampieri C, Mugianesi E, Centurelli M, Franceschi C, Paolisso G: Polymorphic variants of insulin-like growth factor I (IGF-I) receptor and phosphoinositide 3-kinase genes affect IGF-I plasma levels and human longevity: cues for an evolutionarily conserved mechanism of life span control. JClinEndocrinolMetab 2003, 88(7):3299.

37. Kojima T, Kamei H, Aizu T, Arai Y, Takayama M, Nakazawa S, Ebihara Y, Inagaki H, Masui Y, Gondo Y, Sakaki Y, Hirose N: Association analysis between longevity in the Japanese population and polymorphic variants of genes involved in insulin and insulinlike growth factor I signaling pathways. ExpGerontol 2004, 39(I I-1 2): 1595.

38. Kuningas M, Magi R, Westendorp RG, Slagboom PE, Remm M, van Heemst D: Haplotypes in the human Foxola and Foxo3a genes; impact on disease and mortality at old age. EurJHumGenet 2007, I 5(3):294.

39. Brenkman $A B$, Burgering $B M$ : FoxO3a eggs on fertility and aging. Trends Mol Med 2003, 9(I I):464-467.

40. Castrillon DH, Miao L, Kollipara R, Horner JW, DePinho RA: Suppression of ovarian follicle activation in mice by the transcription factor Foxo3a. Science 2003, 30 I(5630):2 I5-2 I 8.

41. Hirschhorn JN, Lohmueller K, Byrne E, Hirschhorn K: A comprehensive review of genetic association studies. Genet Med 2002 4(2):45-61.

42. Bathum L, Christiansen L, Tan Q, Vaupel J, Jeune B, Christensen K: No evidence for an association between extreme longevity and microsomal transfer protein polymorphisms in a longitudinal study of $165 \mathrm{I}$ nonagenarians. EurJHumGenet 2005, I3( I 0): I I 54.

43. Beekman M, Blauw GJ, Houwing-Duistermaat JJ, Brandt BW, Westendorp RG, Slagboom PE: Chromosome 4q25, microsomal transfer protein gene, and human longevity: novel data and a meta-analysis of association studies. JGerontolA BiolSciMedSci 2006, 6 I (4):355.

44. Sild M, Koca C, Bendixen MH, Frederiksen H, McGue M, Kolvraa S, Christensen K, Nexo B: Possible associations between successful aging and polymorphic markers in the Werner gene region. AnnNYAcadSci 2006, 1067:309.

45. Kuro-o M, Matsumura $Y$, Aizawa $H$, Kawaguchi $H$, Suga $T$, Utsugi $T$, Ohyama Y, Kurabayashi M, Kaname T, Kume E, Iwasaki H, lida A, Shiraki-lida T, Nishikawa S, Nagai R, Nabeshima YI: Mutation of the mouse klotho gene leads to a syndrome resembling ageing. Nature 1997, 390(6655):45.

46. Kurosu H, Yamamoto M, Clark JD, Pastor JV, Nandi A, Gurnani P, McGuinness OP, Chikuda H, Yamaguchi M, Kawaguchi H, Shimomura I, Takayama Y, Herz J, Kahn CR, Rosenblatt KP, Kuro-o M: Suppression of aging in mice by the hormone Klotho. Science 2005, 309(5742): 1829

47. Masuda H, Chikuda H, Suga $T$, Kawaguchi $H$, Kuro O: Regulation of multiple ageing-like phenotypes by inducible klotho gene expression in klotho mutant mice. MechAgeing Dev 2005, I26(I2): 1274 .
Publish with Biomed Central and every scientist can read your work free of charge

"BioMed Central will be the most significant development for disseminating the results of biomedical research in our lifetime. "

Sir Paul Nurse, Cancer Research UK

Your research papers will be:

- available free of charge to the entire biomedical community

- peer reviewed and published immediately upon acceptance

- cited in PubMed and archived on PubMed Central

- yours - you keep the copyright 